\title{
Genetic structure of the population with rheumatoid arthritis in north east England: a genetic approach to define different subtypes
}

\author{
S S PAPIHA, ${ }^{1}$ J S LANCHBURY,${ }^{1}$ AND B PAL ${ }^{2}$ \\ From the 'Department of Human Genetics, Medical School, University of Newcastle upon Tyne; and the \\ ${ }^{2}$ Department of Rheumatology, Dryburn Hospital, Durham
}

SUMMARY Clinically and immunologically rheumatoid arthritis (RA) is possibly a heterogeneous disorder. Despite numerous efforts clearer definition of this heterogeneity has been of limited success. Measurements of rheumatoid factor (RF) and antinuclear antibodies (ANA) by conventional methods define subpopulations of patients with RA and in a few recent studies an association of human leucocyte antigens (HLA) undoubtedly indicates the immunogenetic differences in the susceptibility of RA patients with different status of autoantibodies. The studies on a few isolated non-HLA genetic markers in RA are controversial. To understand the role of genetic factors in susceptibility 24 single gene characters other than HLA were investigated in 225 patients with RA classified by humoral status (presence or absence of RF and ANA) into three groups and in 104 healthy control individuals from the north east of England. Locus by locus comparison suggested associations of $\mathrm{MN}$, Lewis, and Bf system with RF positive patients. Although the associations with $\mathrm{MN}$ and Lewis blood groups require further investigations, the involvement of the $\mathrm{Bf}$ locus is in agreement with the immunological component of the disease suggested by HLA associations and it could be due to the phenomenon of linkage disequilibrium. Measures of genetic distance applied to the subpopulations of patients with RA, divided according to the presence or absence of humoral factors, suggest that $\mathrm{RF}^{+} \mathrm{ANA}^{+}, \mathrm{RF}^{-} \mathrm{ANA}^{-}$, and $\mathrm{RF}^{+} \mathrm{ANA}{ }^{-}$subgroups are distinct genetic diseases, each affecting a different subsection of the population which is genetically distinct. Such genetic heterogeneity may suggest a different pathogenetic mechanism for each of these subpopulations of rheumatoid arthritis.

Key words: blood groups, serum proteins, red cell enzymes, phenotypic variation, genetic heterogeneity.

Present evidence that genetic factors may play an important part in the development of rheumatoid arthritis (RA) comes from twin studies, pedigree analysis, and associations with it of marker variants in genetic polymorphic systems. ${ }^{1-7}$ The initial investigations on monozygotic cotwins showed an incidence of the disease 33 times that expected, and family studies showed a relative risk ranging between 1 and $15 \cdot 2$ for the first degree relatives. These

Accepted for publication 21 April 1986.

Correspondence to $\mathrm{Dr} S \mathrm{~S}$ Papiha, Department of Human Genetics, 19 Claremont Place, Newcastle upon Tyne NE2 4AA observations suggested strong genetic susceptibility to the disease.

In the last two decades a few retrospective analyses of the literature cast doubt on the validity of the existing data on familial aggregation of RA, and suggested a weak genetic element in the aetiology of the disease. ${ }^{8}$ Meanwhile, research on its association with human leucocyte antigens once again drew attention to the possibility of genetic predisposition coded at or near the HLA-D locus situated on chromosome 6 in man, or a defective immune response gene which may be in linkage disequilibrium with the HLA-D locus. ${ }^{4}{ }^{10-12}$ 
One of the fundamental reasons for the inconsistency of these lines of evidence-on the one hand, a strong genetic element suggested by the association with B lymphocyte alloantigens, and on the other, a weaker genetic contribution indicated by the family studies-may be the disease heterogeneity, which was perhaps not taken sufficiently into consideration in the initial investigations. Several observations in man clearly indicate that different genetic factors may be involved in different types of rheumatoid arthritis. ${ }^{12} \mathrm{~A}$ strong association between HLA-DR4 and seropositive (rheumatoid factor; $\mathrm{RF}^{+}$) rheumatoid arthritis patients is established in white Europeans, whereas in contrast the frequency of DR4 in seronegative $\left(\mathrm{RF}^{-}\right)$patients is similar to that of the control. ${ }^{13}$ These HLA results in man, though there is some lack of consistency, are supported by experimental studies in certain mammals, ${ }^{14}$ and overall they indicate that seronegative and seropositive patients may be two different genetic subsections of the disease population, with different pathogenetic mechanisms bringing about phenotypically similar diseases.

Another autoantibody frequently encountered in patients with RA is associated with nuclear antigen. The extent of genetic control of antinuclear antibodies (ANA) is not clearly understood, but their association with 'autoimmune' disease suggests that they may play an integral part in the development of the disease. ${ }^{15}$ Once again, depending on the presence or absence of ANA, two populations of patients with RA can be distinguished. The interaction of those gene(s) responsible for the production of the autoantibodies may influence the course and severity of the RA. ${ }^{16} 17$

The aim of the present study, therefore, was to apply available genetic methods to inquire if the patients of different humoral status represent different populations of the same disease. Since the phenotypic expression of the disease will depend on the interactions within the total genotype of the patients the approach here followed was to investigate a large number of single gene markers in systems other than HLA in patient groups of different humoral status, and summarise these data in a single measure of genetic heterogeneity.

\section{Patients and methods}

PATIENT AND CONTROLS

A random collection of 225 adult patients fulfilling the criteria of the American Rheumatism Association for the classification of rheumatoid arthritis was included in this study. ${ }^{18}$ All the patients were white and from the north east of England. They had been followed up in outpatient clinics for three to 30 years. Eighty per cent of the total patient population were over 50 years of age and $40 \%$ over 60 years. The control group of 104 individuals was matched proportionately for sex and region and was mainly drawn from the electoral register.

EXPERIMENTA L PROCEDURE

Five millilitres of blood was obtained from each patient and control by venepuncture, into ethylenediaminetetra-acetate (EDTA) tubes. The samples were sent to the laboratory within four hours of collection. The plasma sample was separated and stored at $-30^{\circ} \mathrm{C}$ for serum protein examination, and the red cells were analysed for blood groups. After washing the red cells with normal saline, lysates were prepared with equal amounts of water for the examination of red cell enzyme.

\section{B L OOD GROUPS}

The red cells were typed using the following antisera: $A_{1}, A_{2}, B, A+B, M, N, S, s, C, c, C^{w}$, $\mathrm{E}, \mathrm{e}, \mathrm{D}, \mathrm{Fy}^{\mathrm{a}}, \mathrm{Fy}^{\mathrm{b}}, \mathrm{P}, \mathrm{K}, \mathrm{k}, \mathrm{Jk}^{\mathrm{a}}, \mathrm{Jk}^{\mathrm{b}}, \mathrm{Lu}^{\mathrm{a}}, \mathrm{Lu}^{\mathrm{b}}, \mathrm{Le}^{\mathrm{a}}$. The tests were carried out by standard serological methods. ${ }^{19}$

\section{SERUM PROTEINS}

The complement components $(\mathrm{C} 3$ and $\mathrm{Bf})$ were analysed immediately after arrival of the sample by high voltage agarose electrophoresis as described by Alper and Propp. ${ }^{20}$ Haptoglobin and transferrin were typed by horizontal starch gel electrophoresis by the method of Smithies. ${ }^{21}$ The group specific components $(\mathrm{Gc})$ were subtyped by isoelectric focusing (IEF) on thin layer polyacrylamide gels as described from this laboratory. ${ }^{22}$

\section{RED CELL ISOENZYMES}

The red cell enzymes adenosine deaminase (ADA), acid phosphatase (AP), phosphoglucomutase (PGM), adenylate kinase (AK), 6-phosphoglucomutase dehydrogenase (6PGD), malate dehydrogenase $(\mathrm{MDH})$, lactate dehydrogenase ( $\mathrm{LDH})$, and superoxide dismutase (SOD) were analysed by horizontal starch gel electrophoresis with the specific conditions given by Harris and Hopkinson, ${ }^{23}$ and the phosphoglucomutase (PGM) subtypes were analysed by IEF on thin layer polyacrylamide gels. ${ }^{22}$

HUMORAL STATUS

The presence of rheumatoid factor was tested by the haemagglutination technique. ${ }^{24} \mathrm{~A}$ serum showing a reaction at a dilution of 1:40 was considered positive. Patients were characterised as seronegative 
if all repeated tests for RF were negative. The presence of antinuclear antibody (IgG) was tested for by an indirect immunofluorescent technique with rat liver. ${ }^{25}$

\section{Results}

Patients were divided into groups depending upon the presence or absence of the humoral factors (RF and ANA). The composition of the groups and the patients tested in each are given in Table 1. Among the total patient group (RA) five subgroups were distinguished: $\mathrm{RF}^{+} \mathrm{ANA}^{-}, \mathrm{RF}^{+} \mathrm{ANA}^{-}, \mathrm{RF}^{+}$ $\mathrm{ANA}^{+}$, and $\mathrm{RF}^{-} \mathrm{ANA}^{-}$.

The phenotype numbers and frequencies for each system (blood groups, red cell enzymes and serum proteins) in the total number of patients with RA, the subgroups, and the controls are listed in Table 2. Tested by $\chi^{2}$, no system shared any deviation of phenotype numbers from the expected. Compared with other surveys of healthy subjects the $P$ blood groups showed slightly aberrant frequencies in the present controls, therefore interpretation of associations if any with this system requires caution. The gene frequencies of the various systems are given in Table 3.

\section{B L OOD GROUPS}

The frequencies of the $A$ and $B$ genes were lower in all the five subgroups than in the control group, but the differences were small and not statistically significant. The absence of $B$ blood group from the seronegative group $\left(\mathrm{RF}^{-} \mathrm{ANA}^{-}\right)$is probably due to the small sample size. The raised $\mathrm{Rh}$ positive frequency observed in other studies ${ }^{26}$ could not be

Table 1 Subgroups of patients with rheumatoid arthritis $(R A)$ depending on the humoral factors

\begin{tabular}{|c|c|c|c|}
\hline $\begin{array}{l}\text { Subgroup } \\
\text { No }\end{array}$ & Subgroup & $\begin{array}{l}\text { Number } \\
\text { tested }\end{array}$ & $\begin{array}{l}\text { Abbreviation } \\
\text { used }\end{array}$ \\
\hline 1 & $\begin{array}{l}\text { Total number of patients } \\
\text { with RA }\end{array}$ & 225 & RA \\
\hline 2 & $\begin{array}{l}\text { Rheumatoid factor positive/ } \\
\text { antinuclcar antibody } \\
\text { negative }\end{array}$ & 165 & $\mathrm{RF}^{+} \mathrm{ANA}^{-}$ \\
\hline 3 & $\begin{array}{l}\text { Rhcumatoid factor positive/ } \\
\text { antinuclear antibody } \\
\text { positive }\end{array}$ & 44 & $\mathrm{RF}^{+} \mathrm{ANA}^{+}$ \\
\hline 4 & $\begin{array}{l}\text { Rheumatoid factor negative/ } \\
\text { antinuclear antibody } \\
\text { ncgative }\end{array}$ & 15 & $\mathrm{RF}^{-} \mathrm{ANA}^{-}$ \\
\hline 5 & Rheumatoid factor positive & 209 & $\mathrm{RF}+$ \\
\hline 6 & $\begin{array}{l}\text { Antinuclear antibodies } \\
\text { negative }\end{array}$ & 180 & $\mathrm{ANA}^{-}$ \\
\hline 7 & $\begin{array}{l}\text { Control (north east } \\
\text { England) }\end{array}$ & 104 & Control \\
\hline
\end{tabular}

One individual $\left(\mathrm{RF}^{-} \mathrm{ANA}^{+}\right)$was excluded from the analysis. confirmed in the present investigation. In the $\mathrm{MN}$ system the $M$ gene showed a significantly raised frequency in the $\mathrm{RF}^{+}$and $\mathrm{RF}^{+} \mathrm{ANA}^{+}$groups $\left(\chi^{2}=4.6\right.$ and $\left.3.85,1 \mathrm{df} ; \mathrm{p}<0.05\right)$ but not in the other subsamples. For Kell, Duffy, Kidd, and Lutheran although appreciable differences existed between certain subgroups of patient and the control, in no system were the differences statistically significant. For the Lewis blood group system the $\mathrm{Le}^{\mathrm{a}+}$ frequencies among the patient groups ranged from $12 \%$ to $23 \%$ compared with $25 \%$ in the controls. The lowest frequency of the $\mathrm{Le}^{\mathrm{a}+}$ antigen was found in the $\mathrm{RF}^{+} \mathrm{ANA}^{+}$group, the difference from the controls being significant (Fisher's exact $p=0 \cdot 05$ ).

\section{SERUM PROTEINS}

For haptoglobin, transferrin, and the $\mathrm{C} 3$ system the difference between each patient group and the control was not significant, though the range of frequency among the patient group for $\mathrm{Hp}^{*} 1$ was appreciable (35-41\%). The suballele frequencies of the Gc system in the control sample were slightly different from those in the north eastern sample reported from this laboratory previously, ${ }^{27}$ but not significantly so $\left(\chi^{2}=6 \cdot 48,4 \mathrm{df} ; \mathrm{NS}\right)$. The overall trend was the same in all RA patient groups; there was an increase of some $5 \%$ in $\mathrm{Gc}^{*} 2$ allele frequency in all the patient groups except the few seronegative patients $\left(\mathrm{RF}^{-} \mathrm{ANA}^{-}\right)$where the increase was slightly less. In the $\mathrm{Bf}$ system all the patient groups showed an increase of between $5 \%$ and $8 \%$ in the $\mathrm{Bf}^{*} \mathrm{~S}$ allele frequency. The individuals with the $\mathrm{Bf}^{*} \mathrm{~S}$ allele overall had a statistically significant high relative risk of developing RA of $1 \cdot 7\left(\chi^{2}=4 \cdot 12,1 \mathrm{df}\right.$; $\mathrm{p}<0.05)$. Although an increased relative risk occurred within each of the patients groups, it was only significant for the $\mathrm{RF}^{+}$and $\mathrm{RF}^{+} \mathrm{ANA}^{-}$groups (Table 4).

\section{RED CELL ENZYMES}

For the red cell enzyme polymorphisms there was a considerable range of gene frequency variation among patient groups, shown for example by the alleles GPT*2 (39-53\%); PGM1*1+ $(60-73 \%)$; $\mathrm{AP}^{*} \mathrm{C}(2-7 \%) ; \operatorname{EsD}^{* 2}(9-17 \%)$, and $\mathrm{ADA}^{*} 2$ $(4-10 \%)$. Overall there was no significant association found between RA and the genes of any enzyme system.

MULTIVARIATE ANALYSIS

Consideration allele by allele of the differences between patients with RA and controls, and among the various RA patient groups, is not very informative in the detection of genetic differences among them. It is by no means unusual that minor differences in a number of features which seem to 
884 Papiha, Lanchbury, Pal

Table 2 Observed phenotype numbers and phenotype frequencies for patients with rheumatoid arthritis and controls

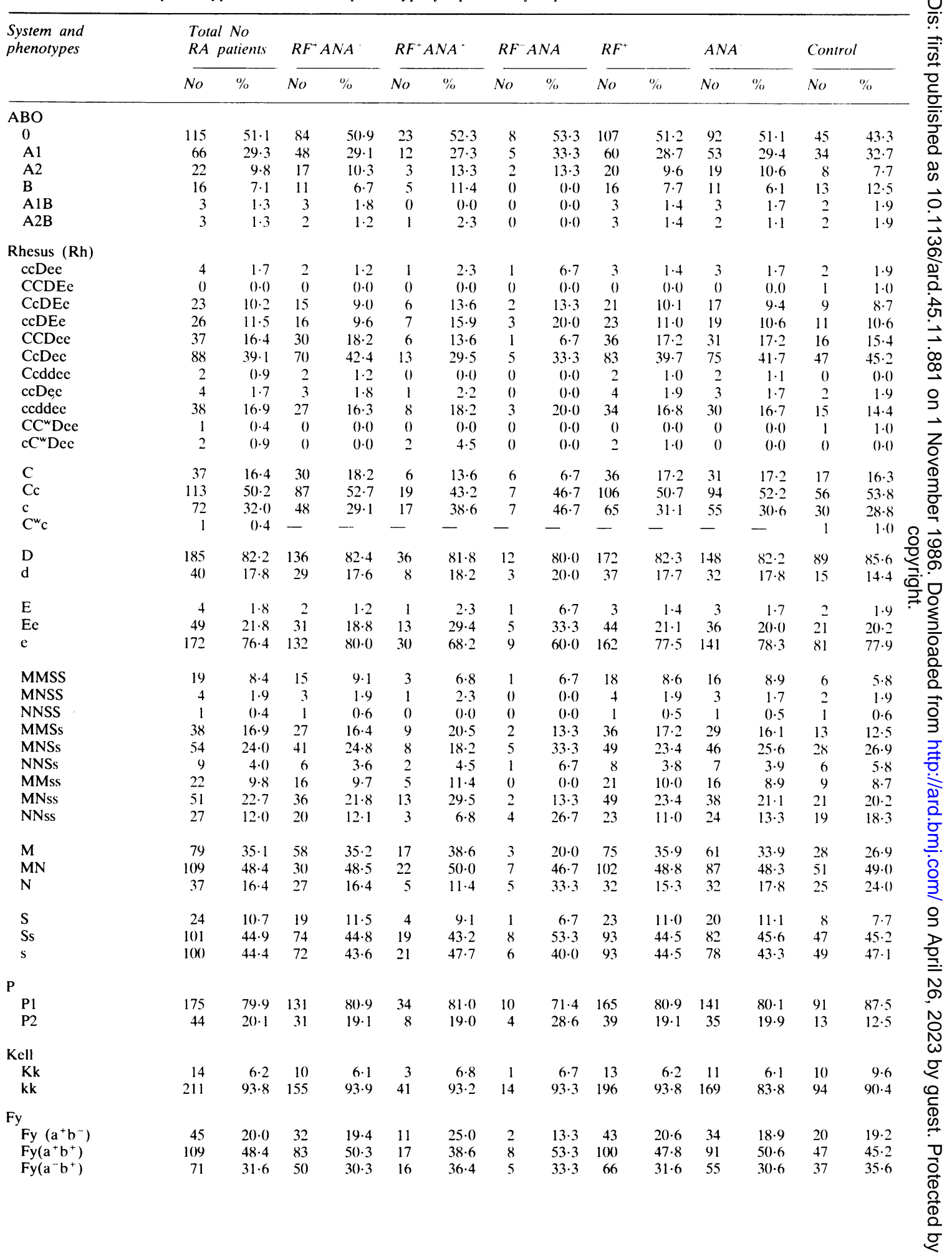




\begin{tabular}{|c|c|c|c|c|c|c|c|c|c|c|c|c|c|c|}
\hline \multirow[t]{2}{*}{$\begin{array}{l}\text { System and } \\
\text { phenotypes }\end{array}$} & \multicolumn{2}{|c|}{$\begin{array}{l}\text { Total No } \\
\text { RA patients }\end{array}$} & \multicolumn{2}{|c|}{$R F^{+} A N A^{-}$} & \multicolumn{2}{|c|}{$R F^{+} A N A^{+}$} & \multicolumn{2}{|c|}{$R F^{-} A N A^{-}$} & \multicolumn{2}{|c|}{$R F^{+}$} & \multicolumn{2}{|c|}{$A N A^{-}$} & \multicolumn{2}{|c|}{ Control } \\
\hline & No & $\%$ & No & $\%$ & No & $\%$ & No & $\%$ & No & $\%$ & No & $\%$ & No & $\%$ \\
\hline \multicolumn{15}{|l|}{ Kidd (Jk) } \\
\hline$J k\left(a^{+} b^{-}\right)$ & 68 & $30 \cdot 2$ & 49 & $29 \cdot 7$ & 14 & $31 \cdot 8$ & 4 & $26 \cdot 7$ & 63 & $30 \cdot 1$ & 53 & $29 \cdot 4$ & 32 & $30 \cdot 8$ \\
\hline $\mathrm{Jk}\left(\mathrm{a}^{+} \mathrm{b}^{+}\right)$ & 106 & $47 \cdot 1$ & 76 & $46 \cdot 1$ & 21 & $47 \cdot 7$ & 9 & $60 \cdot 0$ & 97 & $46 \cdot 4$ & 85 & $47 \cdot 2$ & 44 & $42 \cdot 8$ \\
\hline$J k\left(a^{-} b^{+}\right)$ & 51 & $22 \cdot 7$ & 40 & $24 \cdot 2$ & 9 & $20 \cdot 5$ & 2 & $13 \cdot 3$ & 49 & 23.4 & 42 & $23 \cdot 3$ & 28 & 26.9 \\
\hline \multicolumn{15}{|c|}{ Lutheran (Lu) } \\
\hline $\operatorname{Lu}\left(a^{+} b^{+}\right)$ & 14 & $6 \cdot 2$ & 9 & $5 \cdot 5$ & 5 & $11 \cdot 4$ & 0 & 0.0 & 14 & $6 \cdot 7$ & 9 & $5 \cdot 0$ & 6 & $5 \cdot 8$ \\
\hline $\operatorname{Lu}\left(a^{-} b^{+}\right)$ & 211 & $93 \cdot 8$ & 156 & $94 \cdot 5$ & 39 & $88 \cdot 6$ & 15 & $100 \cdot 0$ & 195 & $93 \cdot 3$ & 171 & $95 \cdot 0$ & 98 & $94 \cdot 2$ \\
\hline \multicolumn{15}{|l|}{ Lewis (Le) } \\
\hline $\operatorname{Le}\left(\mathrm{a}^{+}\right)$ & 45 & $20 \cdot 2$ & 38 & $23 \cdot 2$ & 5 & $11 \cdot 7$ & 2 & $13 \cdot 3$ & 43 & $20 \cdot 8$ & 40 & $22 \cdot 3$ & 26 & $25 \cdot 0$ \\
\hline $\operatorname{Le}\left(a^{-}\right)$ & 96 & $43 \cdot 0$ & 73 & $44 \cdot 5$ & 18 & $41 \cdot 9$ & 4 & $26 \cdot 7$ & 91 & $44 \cdot 0$ & 77 & $43 \cdot 0$ & 68 & $65 \cdot 4$ \\
\hline \multicolumn{15}{|c|}{ Haptoglobin (HP) } \\
\hline 1 & 30 & $13 \cdot 5$ & 20 & $12 \cdot 1$ & 6 & $14 \cdot 0$ & 3 & $20 \cdot 0$ & 26 & $12 \cdot 6$ & 23 & $12 \cdot 8$ & 14 & $13 \cdot 7$ \\
\hline $1-2$ & 106 & $47 \cdot 5$ & 76 & $46 \cdot 1$ & 24 & $55 \cdot 8$ & 6 & $40 \cdot 0$ & 100 & $48 \cdot 3$ & 82 & $45 \cdot 8$ & 55 & $53 \cdot 7$ \\
\hline 2 & 87 & $39 \cdot 0$ & 68 & $41 \cdot 2$ & 13 & $30 \cdot 2$ & 6 & $40 \cdot 0$ & 81 & $39 \cdot 1$ & 74 & $41 \cdot 3$ & 33 & $32 \cdot 4$ \\
\hline \multicolumn{15}{|c|}{ Transferrin (TF) } \\
\hline C & 223 & $99 \cdot 6$ & 164 & $99 \cdot 4$ & 43 & $100 \cdot 0$ & 15 & $100 \cdot 0$ & 207 & $99 \cdot 5$ & 179 & $99 \cdot 4$ & 103 & 99.0 \\
\hline CB & 1 & 0.4 & 1 & 0.6 & 0 & $0 \cdot 0$ & 0 & 0.0 & 1 & 0.5 & 1 & 0.6 & 1 & $1 \cdot 0$ \\
\hline
\end{tabular}

Complement component 3 (C3)

\begin{tabular}{|c|c|c|c|c|c|c|c|c|c|c|c|c|c|c|}
\hline $\mathbf{S}$ & 140 & $62 \cdot 5$ & 102 & $6 \cdot 2$ & 28 & 63.6 & 10 & $66 \cdot 7$ & 130 & $62 \cdot 5$ & 112 & $62 \cdot 6$ & 64 & $61 \cdot 5$ \\
\hline FS & 70 & $31 \cdot 3$ & 53 & $32 \cdot 3$ & 12 & $27 \cdot 3$ & 4 & $26 \cdot 7$ & 65 & $31 \cdot 1$ & 57 & $31 \cdot 8$ & 32 & 30.8 \\
\hline $\mathbf{F}$ & 10 & 4.5 & 6 & $3 \cdot 7$ & 3 & 6.8 & 1 & 6.7 & 9 & $4 \cdot 3$ & 7 & 3.9 & 4 & 3.8 \\
\hline SS096 & 1 & 0.4 & 1 & $0 \cdot 6$ & 0 & 0.0 & 0 & 0.0 & 1 & 0.5 & 1 & 0.6 & 1 & 1.0 \\
\hline FS096 & 1 & 0.4 & 1 & $0 \cdot 6$ & 0 & $0 \cdot 0$ & 0 & 0.0 & 1 & 0.5 & 1 & $0 \cdot 6$ & 0 & 0.0 \\
\hline F107S & 2 & 0.9 & 1 & $0 \cdot 6$ & 1 & $2 \cdot 3$ & 0 & 0.0 & 2 & 1.0 & 1 & $0 \cdot 6$ & 2 & 1.9 \\
\hline SS094 & 0 & 0.0 & 0 & $0 \cdot 0$ & 0 & 0.0 & 0 & 0.0 & 0 & 0.0 & 0 & $0 \cdot 0$ & 1 & 1.0 \\
\hline
\end{tabular}

Properdin factor B (BF)

\begin{tabular}{|c|c|c|c|c|c|c|c|c|c|c|c|c|c|c|}
\hline S & 165 & $73 \cdot 3$ & 123 & 74.5 & 31 & $70 \cdot 5$ & 10 & $66 \cdot 7$ & 154 & $73 \cdot 7$ & 133 & 73.9 & 63 & $61 \cdot 2$ \\
\hline FS & 50 & $22 \cdot 2$ & 36 & $21 \cdot 8$ & 10 & $22 \cdot 7$ & 4 & $26 \cdot 7$ & 46 & $22 \cdot 0$ & 40 & $22 \cdot 2$ & 29 & $28 \cdot 2$ \\
\hline $\mathbf{F}$ & 4 & 1.8 & 3 & 1.8 & 1 & $2 \cdot 3$ & 0 & $0 \cdot 0$ & 4 & 1.9 & 3 & $1 \cdot 7$ & 6 & $5 \cdot 8$ \\
\hline SS07 & 4 & 1.8 & 2 & 1.2 & 2 & $4 \cdot 5$ & 0 & 0.0 & 4 & 1.9 & 2 & $1 \cdot 1$ & 2 & $1 \cdot 1$ \\
\hline F1S & 2 & 1.8 & 1 & 0.6 & 0 & 0.0 & 1 & $6 \cdot 7$ & 1 & 0.5 & 2 & $1 \cdot 1$ & 3 & 2.9 \\
\hline
\end{tabular}

Group specific component (GC)

\begin{tabular}{|c|c|c|c|c|c|c|c|c|c|c|c|c|c|c|}
\hline $1 S$ & 62 & $28 \cdot 1$ & 44 & $27 \cdot 0$ & 14 & 33.8 & 4 & $26 \cdot 7$ & 58 & $28 \cdot 3$ & 48 & $27 \cdot 0$ & 29 & $28 \cdot 7$ \\
\hline 1F1S & 37 & $16 \cdot 7$ & 30 & 18.4 & 4 & 9.5 & 3 & $20 \cdot 0$ & 34 & $16 \cdot 6$ & 33 & 18.5 & 24 & $23 \cdot 8$ \\
\hline $1 \mathrm{~F}$ & 1 & 0.5 & 1 & 0.6 & 0 & 0.0 & 0 & 0.0 & 1 & 0.5 & 0 & 0.6 & 1 & 1.0 \\
\hline 1S2 & 76 & 34.4 & 56 & 34.4 & 15 & $35 \cdot 7$ & 5 & $33 \cdot 3$ & 71 & $34 \cdot 6$ & 61 & $34 \cdot 3$ & 26 & $25 \cdot 7$ \\
\hline $1 F 2$ & 20 & 9.0 & 12 & $7 \cdot 4$ & 6 & $13 \cdot 6$ & 2 & $13 \cdot 3$ & 18 & 8.8 & 14 & 7.9 & 12 & 11.9 \\
\hline 2 & 25 & $11 \cdot 3$ & 20 & $12 \cdot 3$ & 3 & $7 \cdot 1$ & 1 & 6.7 & 23 & $11 \cdot 2$ & 21 & 11.8 & 9 & 8. \\
\hline
\end{tabular}

Glutamic-pyruvate transminase (GPT)

\begin{tabular}{|c|c|c|c|c|c|c|c|c|c|c|c|c|c|c|}
\hline 1 & 38 & $19 \cdot 7$ & 27 & 18.8 & 8 & $20 \cdot 5$ & 3 & $33 \cdot 3$ & 35 & $19 \cdot 1$ & 30 & $19 \cdot 6$ & 24 & \\
\hline $1-2$ & 106 & 54.9 & 80 & 55.6 & 21 & 53.8 & 5 & $55 \cdot 6$ & 101 & $55 \cdot 2$ & 85 & $55 \cdot 6$ & 51 & 55.4 \\
\hline 2 & 48 & 24.9 & 36 & $25 \cdot 0$ & 10 & $25 \cdot 6$ & 1 & $11 \cdot 1$ & 46 & $25 \cdot 1$ & 37 & $24 \cdot 2$ & 16 & 17.4 \\
\hline $1-3$ & 0 & 0.0 & 0 & 0.0 & 0 & $0 \cdot 0$ & 0 & 0.0 & 0 & 0.0 & 0 & 0.0 & 1 & 1.1 \\
\hline $2-3$ & 1 & 0.5 & 1 & 0.7 & 0 & 0.0 & 0 & 0.0 & 1 & 0.5 & 1 & 0.7 & 0 & 0.0 \\
\hline
\end{tabular}

Adenosine deaminase (ADA)

\begin{tabular}{|c|c|c|c|c|c|c|c|c|c|c|c|c|c|c|}
\hline 1 & 204 & 90.7 & 153 & 92.7 & 38 & $86 \cdot 4$ & 12 & $80 \cdot 0$ & 191 & 91.4 & 165 & 91.7 & 92 & 88.5 \\
\hline $1-2$ & 20 & 8.9 & 12 & $7 \cdot 3$ & 5 & 11.4 & 3 & $20 \cdot 0$ & 17 & $8 \cdot 1$ & 15 & $8 \cdot 3$ & 12 & 11.5 \\
\hline 2 & 1 & 0.4 & 0 & 0.0 & 1 & $2 \cdot 3$ & 0 & $0 \cdot 0$ & 1 & 0.5 & 0 & $0 \cdot 0$ & 0 & 0.0 \\
\hline 12 & & & & & & & & & & & & & & \\
\hline $1-2$ & $\begin{array}{r}178 \\
44\end{array}$ & $\begin{array}{l}79 \cdot 1 \\
19.6\end{array}$ & $\begin{array}{r}134 \\
30\end{array}$ & $\begin{array}{l}81 \cdot 2 \\
18 \cdot 2\end{array}$ & $\begin{array}{l}32 \\
11\end{array}$ & $\begin{array}{l}72 \cdot 7 \\
25 \cdot 0\end{array}$ & $\begin{array}{r}11 \\
3\end{array}$ & $\begin{array}{l}73 \cdot 3 \\
20 \cdot 0\end{array}$ & $\begin{array}{r}166 \\
41\end{array}$ & $\begin{array}{l}79.4 \\
19.6\end{array}$ & $\begin{array}{r}145 \\
33\end{array}$ & $\begin{array}{l}80 \cdot 6 \\
18.3\end{array}$ & $\begin{array}{l}79 \\
23\end{array}$ & $\begin{array}{l}76 . \\
22 .\end{array}$ \\
\hline 2 & 3 & 1.3 & 1 & 0.6 & 1 & $2 \cdot 3$ & 1 & 6.7 & 2 & 1.0 & נJ & 1.1 & $2 J$ & \\
\hline
\end{tabular}




\begin{tabular}{|c|c|c|c|c|c|c|c|c|c|c|c|c|c|c|}
\hline \multirow[t]{2}{*}{$\begin{array}{l}\text { System and } \\
\text { phenotypes }\end{array}$} & \multicolumn{2}{|c|}{$\begin{array}{l}\text { Total No } \\
R A \text { patients }\end{array}$} & \multicolumn{2}{|c|}{$R F^{+} A N A^{-}$} & \multicolumn{2}{|c|}{$R F^{+} A N A^{+}$} & \multicolumn{2}{|c|}{$R F A N A^{-}$} & \multicolumn{2}{|c|}{$R F^{+}$} & \multicolumn{2}{|c|}{$A N A$} & \multicolumn{2}{|c|}{ Control } \\
\hline & No & $\%$ & No & $\%$ & No & $\%$ & No & $\%$ & No & $\%$ & No & $\%$ & No & $\%$ \\
\hline \multicolumn{15}{|c|}{ Phosphoglucomutase 1 (PGM) } \\
\hline $1^{+}$ & 98 & $43 \cdot 6$ & 74 & $44 \cdot 8$ & 16 & $36 \cdot 4$ & 8 & $53 \cdot 3$ & 90 & $43 \cdot 1$ & 82 & $45 \cdot 6$ & 38 & $36 \cdot 5$ \\
\hline $1^{+} 1^{-}$ & 34 & $15 \cdot 1$ & 22 & $13 \cdot 3$ & 10 & $22 \cdot 7$ & 2 & $13 \cdot 3$ & 32 & $15 \cdot 3$ & 24 & $13 \cdot 3$ & 18 & $17 \cdot 3$ \\
\hline $1^{-}$ & 2 & 0.9 & 0 & 0.0 & 2 & $4 \cdot 5$ & 0 & $0 \cdot 0$ & 2 & $1 \cdot 0$ & 0 & $0 \cdot 0$ & 1 & $1 \cdot 0$ \\
\hline $2^{+}$ & 8 & $3 \cdot 6$ & 6 & $3 \cdot 6$ & 2 & $4 \cdot 5$ & 0 & $0 \cdot 0$ & 8 & $3 \cdot 8$ & 6 & $3 \cdot 3$ & 9 & $8 \cdot 7$ \\
\hline $2^{+} 2^{-}$ & 3 & $1 \cdot 3$ & 2 & $1 \cdot 2$ & 1 & $2 \cdot 3$ & 0 & $0 \cdot 0$ & 3 & 1.4 & 2 & $1 \cdot 1$ & 2 & 1.9 \\
\hline $1^{+} 2^{+}$ & 53 & $23 \cdot 6$ & 40 & $24 \cdot 2$ & 10 & $22 \cdot 7$ & 2 & $13 \cdot 3$ & 50 & 25.9 & 42 & $23 \cdot 3$ & 23 & $22 \cdot 1$ \\
\hline $1^{-} 2^{+}$ & 9 & $4 \cdot 0$ & 6 & $3 \cdot 6$ & 2 & $4 \cdot 5$ & 1 & $6 \cdot 7$ & 8 & $3 \cdot 8$ & 7 & 3.9 & 2 & 1.9 \\
\hline $1^{-} 2^{-}$ & 1 & $0 \cdot 4$ & 1 & $0 \cdot 6$ & 0 & $0 \cdot 0$ & 0 & $0 \cdot 0$ & 1 & $0 \cdot 5$ & 1 & $0 \cdot 6$ & 2 & 1.9 \\
\hline $1^{+} 2^{-}$ & 17 & $7 \cdot 6$ & 14 & $8 \cdot 5$ & 1 & $2 \cdot 3$ & 2 & $13 \cdot 3$ & 15 & $7 \cdot 2$ & 16 & 8.9 & 8 & $7 \cdot 7$ \\
\hline $2^{-}$ & 0 & $0 \cdot 0$ & 0 & $0 \cdot 0$ & 0 & $0 \cdot 0$ & 0 & $0 \cdot 0$ & 0 & $0 \cdot 0$ & 0 & $0 \cdot 0$ & 1 & $1 \cdot 0$ \\
\hline \multicolumn{15}{|c|}{ Acid phosphatase (AP) } \\
\hline A & 31 & $13 \cdot 8$ & 23 & $13 \cdot 9$ & 6 & $13 \cdot 6$ & 2 & $13 \cdot 3$ & 29 & $13 \cdot 9$ & 25 & $13 \cdot 9$ & 15 & $14 \cdot 4$ \\
\hline BA & 81 & $3 \cdot 6$ & 56 & 33.9 & 19 & $43 \cdot 2$ & 6 & $40 \cdot 0$ & 75 & $35 \cdot 9$ & 62 & $34 \cdot 4$ & 40 & $38 \cdot 5$ \\
\hline B & 88 & $39 \cdot 1$ & 65 & $39 \cdot 4$ & 17 & $38 \cdot 6$ & 5 & $33 \cdot 3$ & 82 & $39 \cdot 2$ & 70 & 38.9 & 42 & $40 \cdot 4$ \\
\hline CB & 19 & $8 \cdot 4$ & 15 & $9 \cdot 1$ & 2 & $4 \cdot 5$ & 2 & $13 \cdot 3$ & 17 & $8 \cdot 1$ & 17 & $9 \cdot 4$ & 6 & $5 \cdot 8$ \\
\hline $\mathrm{CA}$ & 6 & $2 \cdot 7$ & 6 & $3 \cdot 6$ & 0 & $0 \cdot 0$ & 0 & $0 \cdot 0$ & 6 & $2 \cdot 9$ & 6 & $3 \cdot 3$ & 1 & $1 \cdot 0$ \\
\hline \multicolumn{15}{|c|}{ Adenylate kinase (AK) } \\
\hline 1 & 210 & $93 \cdot 3$ & 155 & 93.9 & 39 & $88 \cdot 6$ & 15 & $100 \cdot 0$ & 194 & $92 \cdot 8$ & 170 & $94 \cdot 4$ & 98 & $94 \cdot 2$ \\
\hline $1-2$ & 15 & $6 \cdot 7$ & 10 & $6 \cdot 1$ & 5 & $11 \cdot 4$ & 0 & $0 \cdot 0$ & 15 & $7 \cdot 2$ & 10 & $5 \cdot 6$ & 6 & $5 \cdot 8$ \\
\hline \multicolumn{15}{|c|}{ Phosphogluconate dehydrogenase (PGD) } \\
\hline A & 216 & $96 \cdot 0$ & 157 & $95 \cdot 2$ & 43 & $97 \cdot 7$ & 15 & $100 \cdot 0$ & 200 & $95 \cdot 7$ & 172 & $95 \cdot 6$ & 101 & $97 \cdot 1$ \\
\hline $\mathrm{CA}$ & 9 & $4 \cdot 0$ & 8 & $4 \cdot 8$ & 1 & $2 \cdot 3$ & 0 & $0 \cdot 0$ & 9 & $4 \cdot 3$ & 8 & $4 \cdot 4$ & 3 & $2 \cdot 9$ \\
\hline
\end{tabular}

$\mathrm{LDH}, \mathrm{MDH}$, and SOD were monomorphic in all groups.

Table 3 Gene frequencies in patients with rheumatoid arthritis and controls

\begin{tabular}{|c|c|c|c|c|c|c|c|}
\hline $\begin{array}{l}\text { System and } \\
\text { allele }\end{array}$ & $\begin{array}{l}R A \text { patients } \\
(n=225)\end{array}$ & $\begin{array}{l}R F^{+} A N A^{-} \\
(n=165)\end{array}$ & $\begin{array}{l}R F^{+} A N A^{+} \\
(n=44)\end{array}$ & $\begin{array}{l}R F^{-} A N A^{-} \\
(n=15)\end{array}$ & $\begin{array}{l}R F^{+} \\
(n=209)\end{array}$ & $\begin{array}{l}A N A^{-} \\
(n=180)\end{array}$ & $\begin{array}{l}\text { Control } \\
(n=104)\end{array}$ \\
\hline \multicolumn{8}{|l|}{$\mathrm{ABO}$} \\
\hline A1 & $0 \cdot 1675$ & $0 \cdot 1687$ & $0 \cdot 1482$ & $0 \cdot 1835$ & $0 \cdot 1644$ & $0 \cdot 1699$ & $0 \cdot 1923$ \\
\hline A2 & 0.0696 & 0.0722 & 0.0556 & 0.0862 & $0 \cdot 0686$ & 0.0733 & 0.0622 \\
\hline B & $0 \cdot 0501$ & 0.0496 & 0.0708 & - & 0.0540 & 0.0454 & 0.0855 \\
\hline $\mathrm{O}$ & 0.7128 & 0.7095 & 0.7254 & 0.7303 & 0.7130 & $0 \cdot 7131$ & 0.6599 \\
\hline \multicolumn{8}{|c|}{ Rhesus (Rh) } \\
\hline $\mathrm{C}^{\mathrm{w}} \mathrm{De}$ & 0.0067 & - & $0 \cdot 0227$ & - & $0 \cdot 0048$ & - & 0.0041 \\
\hline $\mathrm{cDe}$ & $0 \cdot 1267$ & $0 \cdot 1061$ & $0 \cdot 1705$ & $0 \cdot 2333$ & $0 \cdot 1196$ & $0 \cdot 1167$ & $0 \cdot 1125$ \\
\hline cde & 0.4270 & 0.4255 & 0.4285 & 0.4667 & 0.4261 & 0.4291 & 0.4181 \\
\hline $\mathrm{C}^{\mathrm{w}} \mathrm{de}$ & - & - & - & - & - & - & 0.0007 \\
\hline $\mathrm{CDe}$ & 0.4082 & 0.4327 & $0 \cdot 3523$ & $0 \cdot 3000$ & 0.4155 & 0.4218 & 0.4298 \\
\hline Cde & 0.0096 & 0.0127 & 0.0000 & 0.0000 & 0.0104 & 0.0116 & 0.0000 \\
\hline CDE & 0.0000 & 0.0000 & 0.0000 & 0.0000 & 0.0000 & 0.0000 & 0.0077 \\
\hline $\mathrm{cDe}$ & 0.0219 & 0.0230 & 0.0260 & - & 0.0237 & 0.0209 & $0 \cdot 0270$ \\
\hline $\mathrm{C}^{\mathrm{w}}$ & 0.0067 & - & 0.0227 & - & $0 \cdot 0048$ & - & 0.0048 \\
\hline $\mathrm{C}$ & 0.4178 & 0.4455 & 0.3523 & $0 \cdot 3000$ & 0.4258 & 0.4333 & 0.4375 \\
\hline c & 0.5756 & 0.5545 & 0.6250 & $0 \cdot 7000$ & $0 \cdot 5694$ & 0.5667 & 0.5577 \\
\hline $\mathrm{D}$ & 0.5784 & 0.5808 & 0.5736 & 0.5528 & 0.5792 & 0.5784 & 0.6202 \\
\hline d & 0.4216 & 0.4192 & 0.4264 & 0.4472 & 0.4208 & 0.4216 & 0.3798 \\
\hline $\mathrm{E}$ & 0.1267 & $0 \cdot 1061$ & $0 \cdot 1705$ & 0.2333 & $0 \cdot 1196$ & $0 \cdot 1167$ & $0 \cdot 1202$ \\
\hline $\mathrm{e}$ & 0.8733 & 0.8939 & 0.8295 & 0.7667 & $0 \cdot 8804$ & 0.883 & 0.8789 \\
\hline
\end{tabular}




\begin{tabular}{|c|c|c|c|c|c|c|c|}
\hline $\begin{array}{l}\text { System and } \\
\text { allele }\end{array}$ & $\begin{array}{l}R A \text { patients } \\
(n=225)\end{array}$ & $\begin{array}{l}R F^{+} A N A^{-} \\
(n=165)\end{array}$ & $\begin{array}{l}R F^{+} A N A^{+} \\
(n=44)\end{array}$ & $\begin{array}{l}R F^{-} A N A^{-} \\
(n=15)\end{array}$ & $\begin{array}{l}R F^{+} \\
(n=209)\end{array}$ & $\begin{array}{l}A N A^{-} \\
(n=180)\end{array}$ & $\begin{array}{l}\text { Control } \\
(n=104)\end{array}$ \\
\hline Ms & $0 \cdot 2820$ & $0 \cdot 2905$ & 0.2544 & 0.2944 & $0 \cdot 2826$ & $0 \cdot 2911$ & $0 \cdot 2484$ \\
\hline Ns & 0.0492 & 0.0489 & 0.0525 & 0.0389 & 0.0499 & 0.0478 & 0.0545 \\
\hline Ms & $0 \cdot 3114$ & $0 \cdot 3034$ & $0 \cdot 3820$ & $0 \cdot 1389$ & 0.3202 & 0.2895 & $0 \cdot 2661$ \\
\hline Ns & 0.3575 & $0 \cdot 3572$ & $0 \cdot 3112$ & 0.5277 & $0 \cdot 3472$ & $0 \cdot 3716$ & 0.4310 \\
\hline $\mathbf{M}$ & 0.5933 & 0.5939 & 0.6364 & 0.4333 & 0.6029 & 0.5806 & 0.5144 \\
\hline $\mathrm{N}$ & 0.4067 & 0.4061 & $0 \cdot 3636$ & 0.5667 & $0 \cdot 3971$ & 0.4194 & 0.4856 \\
\hline $\mathbf{S}$ & 0.3311 & $0 \cdot 3394$ & 0.3068 & $0 \cdot 3333$ & 0.3325 & 0.3389 & 0.3029 \\
\hline $\mathbf{s}$ & 0.6689 & $0 \cdot 6606$ & 0.6932 & $0 \cdot 6667$ & $0 \cdot 6675$ & 0.6611 & 0.6971 \\
\hline $\mathbf{P}$ & $(n=219)$ & $(n=165)$ & $(n=42)$ & $(n=14)$ & $(n=204)$ & $(n=180)$ & $(n=104)$ \\
\hline 1 & $0 \cdot 5518$ & 0.5626 & 0.5636 & 0.4655 & 0.5628 & 0.5541 & 0.6465 \\
\hline 2 & 0.4482 & 0.4374 & 0.4364 & 0.5345 & 0.4372 & 0.4459 & 0.3535 \\
\hline Kell (K) & $(n=225)$ & $(n=165)$ & $(n=44)$ & $(n=15)$ & $(n=209)$ & $(n=180)$ & $(n=104)$ \\
\hline K & 0.0311 & 0.0303 & 0.0341 & 0.0333 & 0.0311 & 0.0306 & 0.0481 \\
\hline $\mathrm{k}$ & 0.9689 & 0.9697 & 0.9659 & 0.9667 & 0.9689 & 0.9694 & 0.9519 \\
\hline \multicolumn{8}{|l|}{$\%$ Duffy (Fy) } \\
\hline Fy $^{\mathrm{a}}$ & 0.4422 & 0.4455 & 0.4432 & 0.4000 & 0.4450 & 0.4417 & 0.4183 \\
\hline $\mathrm{Fy}^{\mathrm{b}}$ & $0 \cdot 5578$ & 0.5545 & $0 \cdot 5568$ & $0 \cdot 6000$ & $0 \cdot 5550$ & 0.5583 & 0.5817 \\
\hline \multicolumn{8}{|l|}{ Kidd (Jk) } \\
\hline $\mathrm{Jk}^{\mathrm{a}}$ & 0.5378 & 0.5273 & 0.5568 & 0.5667 & $0.5 \overline{3} 35$ & 0.5306 & 0.5192 \\
\hline $\mathbf{J k}^{\mathrm{b}}$ & 0.4622 & 0.4727 & 0.4432 & 0.4333 & 0.4665 & 0.4694 & 0.4808 \\
\hline \multicolumn{8}{|l|}{ Lutheran (Lu) } \\
\hline $\mathrm{Lu}^{\mathrm{a}}$ & 0.0311 & 0.0273 & 0.0568 & - & 0.0335 & 0.0250 & 0.0288 \\
\hline $\mathbf{L u}^{\mathbf{b}}$ & 0.9689 & 0.9727 & 0.9432 & $1 \cdot 000$ & 0.9664 & 0.9750 & 0.9712 \\
\hline Lewis (Le) & $(n=223)$ & $(n=164)$ & $(n=43)$ & $(n=15)$ & $(n=207)$ & $(n=179)$ & $(n=104)$ \\
\hline Le $a+(\%)$ & $20 \cdot 2$ & $23 \cdot 2$ & $11 \cdot 6$ & $40 \cdot 0$ & $20 \cdot 8$ & $22 \cdot 3$ & $25 \cdot 0$ \\
\hline \multicolumn{8}{|l|}{ Haptoglobin (HP) } \\
\hline 1 & $0 \cdot 3722$ & $0 \cdot 3537$ & 0.4186 & 0.4000 & 0.3671 & 0.3575 & 0.4069 \\
\hline 2 & 0.6278 & $0 \cdot 6463$ & 0.5814 & 0.6000 & $0 \cdot 6328$ & 0.6425 & 0.5931 \\
\hline Transferrin (TF) & $(n=224)$ & $(n=165)$ & $(n=43)$ & $(n=15)$ & $(n=208)$ & $(n=180)$ & $(n=104)$ \\
\hline C & 0.9978 & 0.9970 & 1.0000 & 1.0000 & 0.9976 & 0.9972 & 0.9952 \\
\hline D & 0.0022 & 0.0030 & - & - & 0.0024 & $0 \cdot 0028$ & 0.0048 \\
\hline Complement component 3 (C3) & $(n=224)$ & $(n=164)$ & $(n=44)$ & $(n=15)$ & $(n=208)$ & $(n=179)$ & $(n=104)$ \\
\hline $\mathrm{S}$ & 0.7880 & 0.7896 & 0.7841 & $0 \cdot 8000$ & 0.7885 & 0.7905 & 0.7885 \\
\hline $\mathbf{F}$ & 0.2031 & $0 \cdot 2012$ & $0 \cdot 2045$ & $0 \cdot 2000$ & $0 \cdot 2019$ & $0 \cdot 2011$ & $0 \cdot 1923$ \\
\hline SO94 & - & - & - & - & - & - & 0.0048 \\
\hline SO96 & $0 \cdot 0045$ & $0 \cdot 0061$ & - & - & $0 \cdot 0048$ & 0.0056 & 0.0048 \\
\hline F107 & 0.0045 & 0.0031 & $0 \cdot 0114$ & - & 0.0048 & 0.0028 & 0.0096 \\
\hline Properdin factor B (BF) & $(n=225)$ & $(n=165)$ & $(n=44)$ & $(n=15)$ & $(n=209)$ & $(n=180)$ & $(n=104)$ \\
\hline $\mathrm{s}$ & $0 \cdot 8578$ & $0 \cdot 8636$ & 0.8409 & 0.8333 & 0.8589 & 0.8611 & 0.7767 \\
\hline $\mathbf{F}$ & $0 \cdot 1289$ & $0 \cdot 1273$ & $0 \cdot 1364$ & $0 \cdot 1333$ & $0 \cdot 1292$ & $0 \cdot 1278$ & $0 \cdot 1990$ \\
\hline SO7 & 0.0089 & 0.0061 & 0.0227 & - & 0.0096 & 0.0055 & 0.0097 \\
\hline F1 & 0.0044 & 0.0030 & - & 0.0333 & 0.0024 & 0.0055 & 0.0146 \\
\hline \multicolumn{8}{|l|}{ Group specific } \\
\hline component (GC) & $(n=221)$ & $(n=163)$ & $(n=42)$ & $(n=15)$ & $(n=205)$ & $(n=178)$ & $(n=101)$ \\
\hline is & 0.5362 & 0.5337 & 0.5595 & 0.5333 & 0.5390 & 0.5337 & 0.5347 \\
\hline $1 F$ & 0.1335 & $0 \cdot 1350$ & $0 \cdot 1190$ & $0 \cdot 1667$ & $0 \cdot 1317$ & $0 \cdot 1376$ & $0 \cdot 1881$ \\
\hline 2 & $0 \cdot 3303$ & $0 \cdot 3313$ & $0 \cdot 3214$ & $0 \cdot 3000$ & $0 \cdot 3293$ & $0 \cdot 3287$ & $0 \cdot 2772$ \\
\hline
\end{tabular}




\begin{tabular}{|c|c|c|c|c|c|c|c|}
\hline $\begin{array}{l}\text { System and } \\
\text { allele }\end{array}$ & $R A$ patients & $R F^{+} A N A^{-}$ & $\mathrm{RF}^{+} \mathrm{ANA}^{+}$ & $R F^{-} A N A^{-}$ & $R F^{+}$ & $A N A^{-}$ & Control \\
\hline \multicolumn{8}{|l|}{ Glutamic-pyruvate } \\
\hline transaminase (GPT) & $(n=193)$ & $(n=144)$ & $(n=39)$ & $(n=9)$ & $(n=183)$ & $(n=153)$ & $(n=92)$ \\
\hline 1 & 0.4715 & 0.4653 & 0.4744 & 0.6111 & 0.4672 & 0.4793 & 0.5435 \\
\hline 2 & 0.5259 & 0.5313 & 0.5256 & $0 \cdot 3889$ & 0.5301 & 0.5229 & 0.4511 \\
\hline 3 & 0.0026 & 0.0035 & 0.0000 & 0.0000 & 0.0027 & 0.0033 & $0 \cdot 0054$ \\
\hline Adenosine deaminase (ADA) & $(n=225)$ & $(n=165)$ & $(n=44)$ & $(n=15)$ & $(n=209)$ & $(n=180)$ & $(n=104)$ \\
\hline 1 & 0.9511 & 0.9636 & 0.9205 & 0.9000 & 0.9545 & 0.9583 & 0.9423 \\
\hline 2 & 0.0489 & 0.0364 & 0.0795 & $0 \cdot 1000$ & 0.0455 & $0 \cdot 0417$ & 0.0577 \\
\hline \multicolumn{8}{|l|}{ Esterase D (EsD) } \\
\hline 1 & 0.8889 & $0 \cdot 9030$ & $0 \cdot 8523$ & 0.8333 & $0 \cdot 8923$ & $0 \cdot 8972$ & $0 \cdot 8702$ \\
\hline 2 & $0 \cdot 1111$ & 0.0970 & $0 \cdot 1477$ & $0 \cdot 1667$ & $0 \cdot 1077$ & $0 \cdot 1028$ & $0 \cdot 1298$ \\
\hline \multicolumn{8}{|l|}{ Phosphoglucomutase 1 (PGM) } \\
\hline $1^{+}$ & 0.6667 & 0.6788 & $0 \cdot 6023$ & 0.7773 & 0.6627 & 0.6833 & $0 \cdot 6010$ \\
\hline $1^{-}$ & $0 \cdot 1067$ & $0 \cdot 0879$ & $0 \cdot 1818$ & $0 \cdot 1000$ & $0 \cdot 1077$ & 0.0889 & $0 \cdot 1154$ \\
\hline $2^{+}$ & $0 \cdot 1800$ & $0 \cdot 1818$ & $0 \cdot 1932$ & $0 \cdot 1000$ & $0 \cdot 1842$ & $0 \cdot 1750$ & $0 \cdot 2163$ \\
\hline $2^{-}$ & 0.0467 & 0.0515 & 0.0227 & $0 \cdot 0667$ & 0.0454 & 0.0528 & 0.0673 \\
\hline \multicolumn{8}{|l|}{ Acid phosphatase (AP) } \\
\hline A & $0 \cdot 3311$ & $0 \cdot 3273$ & $0 \cdot 3523$ & $0 \cdot 3333$ & 0.3325 & $0 \cdot 3278$ & $0 \cdot 3413$ \\
\hline B & $0 \cdot 6133$ & 0.6091 & 0.6250 & $0 \cdot 6000$ & 0.6124 & 0.6083 & 0.6250 \\
\hline $\mathrm{C}$ & 0.556 & 0.636 & 0.0227 & 0.0667 & 0.0550 & 0.0639 & 0.0337 \\
\hline \multicolumn{8}{|l|}{ Adenylate kinase (AK) } \\
\hline 1 & 0.9667 & 0.9697 & 0.9432 & $1 \cdot 0000$ & 0.9641 & 0.9722 & 0.9712 \\
\hline 2 & 0.0333 & 0.0303 & 0.0568 & - & $0 \cdot 0359$ & 0.0278 & $0 \cdot 0288$ \\
\hline \multicolumn{8}{|l|}{$\begin{array}{l}\text { Phosphogluconate } \\
\text { dehydrogenase (PGD) }\end{array}$} \\
\hline $\mathrm{C}$ & 0.9800 & 0.9758 & 0.9886 & 1.0000 & 0.9785 & 0.9778 & 0.9856 \\
\hline A & 0.0200 & 0.0242 & 0.0114 & - & $0 \cdot 0215$ & 0.0222 & 0.0144 \\
\hline
\end{tabular}

Table 4 Association of Bf system with rheumatoid arthritis

\begin{tabular}{|c|c|c|c|c|c|c|}
\hline \multicolumn{2}{|c|}{ Group comparison } & \multirow{2}{*}{$\begin{array}{c}B f^{*} S / p o s \\
165 \\
63\end{array}$} & \multirow{2}{*}{$\begin{array}{l}B f^{*} \text { S/neg } \\
54 \\
35\end{array}$} & \multirow{2}{*}{$\frac{R R}{1 \cdot 7}$} & \multirow{2}{*}{$\frac{\chi^{2}}{4 \cdot 12}$} & \multirow{2}{*}{$\begin{array}{l}p \text { Value } \\
<0.05\end{array}$} \\
\hline 1 & $\begin{array}{l}\text { RA patient } \\
\text { Control }\end{array}$ & & & & & \\
\hline 2 & $\begin{array}{l}\text { RF }^{+} \text {ANA }^{-} \text {patient } \\
\text { Control }\end{array}$ & $\begin{array}{r}123 \\
63\end{array}$ & $\begin{array}{l}39 \\
35\end{array}$ & $1 \cdot 8$ & $4 \cdot 05$ & $<0.05$ \\
\hline 3 & $\begin{array}{l}\mathrm{RF}^{+} \mathrm{ANA}^{+} \text {patient } \\
\text { Control }\end{array}$ & $\begin{array}{l}31 \\
63\end{array}$ & $\begin{array}{r}1 \\
35\end{array}$ & $1 \cdot 6$ & $1 \cdot 2$ & $>0.05$ \\
\hline 4 & $\begin{array}{l}\mathrm{RF}^{-} \mathrm{ANA}^{-} \text {patient } \\
\text { Control }\end{array}$ & $\begin{array}{l}10 \\
63\end{array}$ & $\begin{array}{r}4 \\
35\end{array}$ & $1 \cdot 4$ & $0 \cdot 50$ & $>0.05$ \\
\hline 5 & $\begin{array}{l}\mathrm{RF}^{+} \text {patient } \\
\text { Control }\end{array}$ & $\begin{array}{r}154 \\
63\end{array}$ & $\begin{array}{l}50 \\
35\end{array}$ & $1 \cdot 7$ & $4 \cdot 08$ & $<0.05$ \\
\hline 6 & $\begin{array}{l}\text { ANA }^{-} \text {patient } \\
\text { Control }\end{array}$ & $\begin{array}{r}133 \\
63\end{array}$ & $\begin{array}{l}43 \\
35\end{array}$ & $1 \cdot 7$ & $3 \cdot 37$ & $>0.05$ \\
\hline
\end{tabular}

$\mathrm{RR}=$ relative risk.

show little discrimination when considered one by one can when combined produced an overall meaningful pattern. For example, the faces of two individuals appear to the human eye quite distinct, yet one would find it very difficult to distinguish between them say by the interorbital distance or upper lip length considered alone. When all characters are combined into an overall picture by the human eye they are seen to be quite different. Such a procedure is paralleled by the statistical techniques of multivariate analysis, and their application is a well established method of assisting the interpretation of genetic characters.

Many measures have been defined of the genetic similarity or dissimilarity among various populations; one group of multivariate measures are genetic distance statistics, another relates to kinship. If patients with different humoral status represent different genetic subsets of the population then such measures may be useful indicators of the genetic heterogeneity of the disease.

Of the groups of multivariate statistics that represent kinship, the index of Harpending and Jenkins ${ }^{28}$ is particularly useful. The Harpending $R$ matrix is based on the mean standardised deviation of gene frequencies in each group from the total mean $\left(r_{i i}\right)$ and on the average covariance of the gene 
frequencies in a pair of subgroups relative to the mean $\left(r_{i j}\right)$. In this way the frequencies of gene $K$ from the subgroups are transformed into a single value matrix of scaled gene frequency covariance, where the $r_{i j}$ element of $R$ matrix is:

$$
r_{i j}=\frac{1}{k} \sum \frac{\left(p_{i k}-\bar{p}_{h}\right)\left(p_{i k}-\tilde{p}_{h}\right)}{\bar{p}_{k}\left(1-\bar{p}_{k}\right)}
$$

where $p_{i k}$ is the frequency of allele $k$ in group $i$ and $\bar{p}_{k}$ is the mean frequency of allele $k$ over all groups. The elements $r_{i i}$ are obtained as:

$$
r_{i i}=\frac{1}{k} \sum \frac{\left(p_{i k}-\tilde{p}_{k}{ }^{2}\right)}{\tilde{p}_{k}\left(1-\tilde{p}_{k}\right)}
$$

Table 5 gives the $\mathrm{R}$ matrix obtained from the gene frequency data of blood groups, serum proteins, and red cell enzymes. Genetic distinction between the control and the rheumatoid population was shown by the consistently negative values of the control group compared with each of the other subsamples. The patients with both humoral factors present showed negative values against the $\mathrm{RF}^{+} \mathrm{ANA}{ }^{-}$and $\mathrm{RF}^{-} \mathrm{ANA}^{-}$subgroups, suggesting the least affinity with these groups. Genetic distance was analysed only on the patient subgroups $\left(\mathrm{RF}^{+} \mathrm{ANA}^{-}\right.$; $\mathrm{RF}^{+} \mathrm{ANA}^{+} ; \mathrm{RF}^{-} \mathrm{ANA}^{-}$) and controls.

The genetic distances $\left(d^{2}\right)$ derived from the $R$ matrix are given in Table 6 . The largest distance of 0.034 was found between the $\mathrm{RF}^{+} \mathrm{ANA}^{+}$and $\mathrm{RF}^{-} \mathrm{ANA}^{-}$subgroups. Other large distances were found between $\mathrm{RF}^{-} \mathrm{ANA}^{-}$and controls, and $\mathrm{RF}^{-} \mathrm{ANA}^{-}$and $\mathrm{RF}^{+} \mathrm{ANA}^{-}$groups $(0.026$ and 0.025 respectively).

The $\mathrm{d}^{2}$ matrices were reduced to two dimensional plots by using the first two eigenvectors in a principal component analysis and a dendrogram based on the maximum linkage method (Figs 1 and 2 respectively). The isolated position in these plots of the $\mathrm{RF}^{-} \mathrm{ANA}^{-}$group is probably partly due to their small sample size, but the genetic distinction of the patients with both humoral factors is beyond doubt.
Table 6 Genetic distance $\left(d^{2}\right)$ between control and $R A$ subgroups

\begin{tabular}{llllll}
\hline Group & & 1 & 2 & 3 & 4 \\
\hline 1 & RF $^{+}$ANA $^{-}$ & - & & & \\
2 & RF $^{+}$NNA $^{+}$ & 0.0131 & - & & \\
3 & FF $^{-}$ANA $^{-}$ & 0.0252 & 0.0336 & - & \\
4 & Control $^{-0.0105}$ & 0.0144 & 0.0256 & - \\
\hline
\end{tabular}

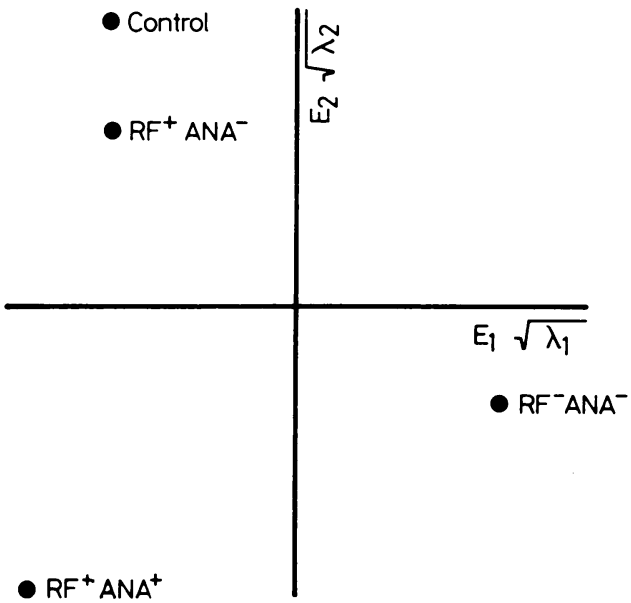

Fig. 1 Eigenvector diagram showing genetic relation of $R A$ subgroups and controls.

\section{Discussion}

As an illustration of the usefulness of polymorphism for understanding genetic heterogeneity and disease aetiology this investigation of the association of genetic markers and rheumatoid arthritis is quite encouraging. It is appreciated that in multiple testing some apparently conventionally significant results may arise by chance, and this may be one interpretation of the findings in the $\mathrm{MN}, \mathrm{Le}^{\mathrm{a}}$, and $\mathrm{Bf}$

\begin{tabular}{|c|c|c|c|c|c|c|c|c|}
\hline Group & No & $\begin{array}{l}1 \\
\text { Total RA } \\
\text { patients }\end{array}$ & $\begin{array}{l}2 \\
R F^{+} A N A^{-}\end{array}$ & $\begin{array}{l}3 \\
R F^{+} A N A^{+}\end{array}$ & ${ }^{4} F^{-} A N A^{-}$ & $\begin{array}{l}5 \\
R F^{+}\end{array}$ & $\begin{array}{l}6 \\
A N A^{-}\end{array}$ & $\begin{array}{l}7 \\
\text { Controls }\end{array}$ \\
\hline Total RA patients & 1 & $0 \cdot 0000$ & & & & & & \\
\hline $\mathrm{RF}^{+} \mathrm{ANA}^{-}$ & 2 & 0.0009 & $0 \cdot 0000$ & & & & & \\
\hline $\mathrm{RF}^{+} \mathrm{ANA}^{+}$ & 3 & 0.0001 & $-0 \cdot 0018$ & 0.0000 & & & & \\
\hline $\mathrm{RF}^{-} \mathrm{ANA}^{-}$ & 4 & -0.0019 & 0.0028 & -0.0046 & 0.0000 & & & \\
\hline $\mathbf{R F}^{+}$ & 5 & $0 \cdot 0007$ & 0.0012 & $0 \cdot 0003$ & $-0 \cdot 0032$ & 0.0000 & & \\
\hline ANA $^{-}$ & 6 & 0.0006 & $0 \cdot 0015$ & $-0 \cdot 0020$ & -0.0012 & 0.0008 & & \\
\hline Controls & 7 & $-0 \cdot 0010$ & -0.0009 & $-0 \cdot 0002$ & -0.0024 & $-0 \cdot 0008$ & $-0 \cdot 0011$ & $0 \cdot 0000$ \\
\hline
\end{tabular}

Table $5 R$ matrix calculated from gene frequency data on control and $R A$ groups for 19 polymorhic systems 


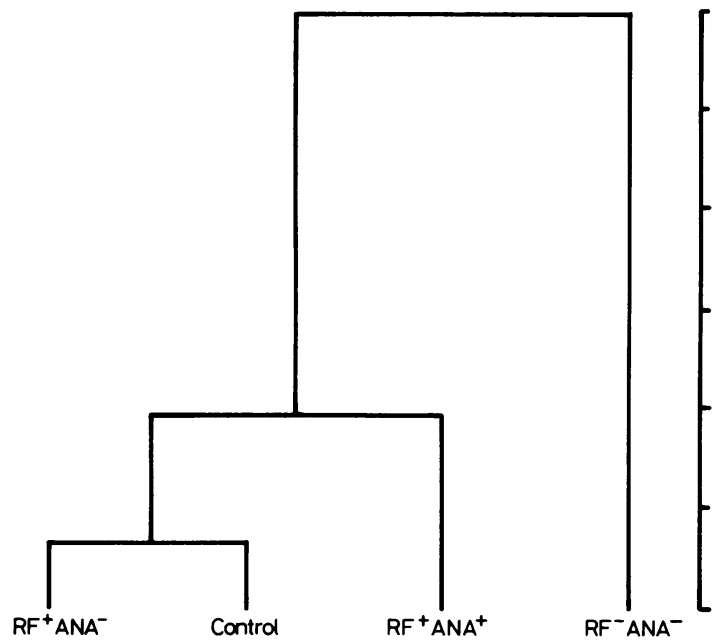

Fig. 2 Dendogram of various $R A$ subgroups and control constructed from Harpending $d^{2}$ matrix.

results. The $p$ values are given, however, because they draw attention to the systems where further investigation may be profitable, but the significance of these individual systems is of little relevance to the general directions shown by the multivariate analysis.

There is marked association with $\mathrm{Bf}$ in the total group of patients with RA and patients with the rheumatoid factor. Other immunogenetic studies suggest an association of HLA-DR4 or an unknown immune response gene in linkage disequilibrium with the $\mathrm{D}$ locus in seropositive $\left(\mathrm{RF}^{+}\right)$rheumatoid arthritis patients. The $\mathrm{Bf}$ gene is also located within the major histocompatibility region, and the association found with it could well suggest an association with an immune response gene through the phenomena of linkage disequilibrium. Futher analysis of extended haplotypes in families with RA may be helpful in predicting the defective immune response and precise genetic susceptibility to rheumatoid arthritis. It will also be useful to include other gene markers, like $\alpha_{1}$ antitrypsin, which are thought to be associated with severe seropositive RA.

Another interesting association seems to be with the Lewis blood group, with a decrease of the Le(a) blood groups in patients with RA in whom both RF and ANA are present. How this Lewis association may be related to the aetiology of disease is not clear, but it seems to be worth further investigation since in another rheumatic disorder, primary Sjögren's syndrome, an association with the Lewis antigen has recently been shown. ${ }^{29}$

Most studies of disease association have been conducted in terms of variation at a single locus. The present investigation shows that genetic distance on approach, which incorporates variation of a large number of loci simultaneously, can be profitably $\stackrel{\vec{S}}{\rightarrow}$ used to examine the genetic heterogeneity of a을 disease. The present results clearly show that seronegative patients $\left(\mathrm{RF}^{-} \mathrm{ANA}^{-}\right)$and patients with both humoral factors $\left(\mathrm{RF}^{+} \mathrm{ANA}^{+}\right)$are two $\stackrel{\mathbb{D}}{\circ}$ distinct entities. These two patient groups represent different subsections of the population, which are genetically distinct. The difference seen in the clinical course of the disease may be the result of interaction of genes controlling the production of $\sigma$ these humoral factors with genetic environmental and physiological factors. Certainly in conclusion it seems that this multivariate analysis shows that rheumatoid arthritis is a genetically heterogeneous disease, and the various subtypes of the disease $\infty_{\infty}^{\infty}$ involve genetically distinct individuals.

We thank Professor D F Roberts for his continuous support in this work and Drs P Mangion. M A Hossain, and I D Griffiths for their Z help and advice. Thanks are also due to Mrs A Stephenson for the statistical help.

\section{References} In: Duthic J J R Alexander W R M eds. Rheumatic diseas Edinburgh: University Press, 1968: 19-28. (Pfizer medie্]! monographs; No. 3)

2 Lawrence J S. Rheumatoid disease in populations. Londomt. Heinemann, 1977.

3 Panayi G S. Woolcy P H. B lymphocytc alloantigens in the $\overline{0}$ study of genetic basis of rheumatoid arthritis. Ann Rheum Dis 1977: 36: 365-8.

4 Stastny P. Rheumatoid arthritis. In: Teresaki P, ed. Histocompatibility testing. Los Angeles: UCLA Press, 1980.

5 Cox D W. Huber O. Association of severe rheumatoid arthritis with heterozygosity of $\alpha_{1}$ antitrypsin deficiency. Clin Genet? 1980: 17: $153-60$.

6 Raum D, Awdeh Z. Glass D, Kammer G, et al Extended:haplotypes of chromosome 6 in adult rheumatoid arthritis. 을 Arthritis Rheum 1984; 27: 516-21.

7 Dahlquist S R. Beckman G. Beckman L. Bf and C3 complement types in rhcumatoid arthritis. Hum Hered 1985; 35: 240-5.

$8 \mathrm{O}^{\circ}$ Brien W M. The genetics of rheumatoid arthritis. Clin Exp Immunol 1967: 2: 785-802.

9 Bennett P H. Some problems in the genetics and epidemiology음 of rhcumatoid arthritis. In: Dumonde D C. ed. Infection and immunology in the rheumatoid diseases. Oxford: Blackwello Scientific, 1976: 301-6.

10) Panayi G S, Woolcy P H. Batchelor J R. HLA DRw4 and rhcumatoid arthritis. Lancet 1979; i: 730 .

11 Woodrow J C. Nichol F E. Zaphiropoulous G. DR antigen andN rhcumatoid arthritis: a study of two populations. Br Med J 1981 : N 283: $1287-8$.

12 Alarcon G S, Koopman W J, Acton R T. Barger B O Seronegative rheumatoid arthritis. A distinct immunogenctic discase? Arthritis Rheum 1982: 25: 502-7.

13 Doublong J H. Forre O. Kass E. Thorsby E. HLA antigens and rheumatoid arthritis: Association between HLA DRw4 positiv-ity and IgM rhcumatoid factor production. Arthritis Rheum 1980; 23: 309-13 
14 Van Snick J L, Coulie P G, Stevens M. Genetic control of rheumatoid factor production in the mouse. Arthritis Rheum 1983; 26:1085-90.

15 Aho K, Sistonen O, Takala J, Sievers K. Genetics of autoantibodies in relation to disease: an epidemiological study of two population areas. Acta Med Scand 1982; 211: 213-8.

16 Masi A T, Maldonado-Cocco J A, Kaplan S B, et al. Prospective study of the early course of rheumatoid arthritis in young adults: comparison of patients with and without rheumatoid factor positivity at entry and identification of variables correlating with outcome. Semin Arthritis Rheum 1976; 4: 299-326.

17 Wiik A, Munthie E. Complement fixing granulocyte specific antinuclear factor in neutropenic cases of rheumatoid arthritis. Immunology 1974; 26: 1127-34.

18 American Rheumatism Association committee. Diagnostic criteria for rheumatoid arthritis 1958. Ann Rheum Dis 1959; 18: 49-53.

19 Mourant A E, Kopec A C, Domaniewska-Sobezak K. The distribution of the human blood group and other polymorphisms. Oxford: Oxford University Press, 1976. (Oxford monograph on medical genetics).

20 Alper C A, Propp R P. Genetic polymorphism of third component of human complement (C3). J Clin Invest 1976: 47: 2181-91.

21 Smithies $\mathrm{O}$. Zone electrophoresis in starch gels: group variation in the serum proteins of normal human adults. Biochem $J$ 1955; 61: 629-41.

22 Papiha S S, White I, Roberts D F. Some genetic implications of isoelectric focusing of human red cell phosphoglucomutase and serum protein group specific component $(\mathrm{Gc})$. Hum Genet 1983; 63: 67-72.

23 Harris H, Hopkinson D A. Handbook of enzyme electrophoresis in human genetics. Amsterdam: North Holland, 1976.

24 Finnie A. Masson M. Muir E. Evaluation of the RANA test for rheumatoid factor. Med Lab Technol 1974; 31: 323-6.

25 Beck J S. Variation in the morphological patterns of autoimmune nuclear fluorescence. Lancet 1961; i: 1203-5.

26 Mourant A E, Kopec A C, Domaniewska-Sobezak K. Blood group and disease. A study of association of disease with blood group and other polymorphisms. Oxford: Oxford University Press, 1978. (Oxford monograph on medical genetics).

27 Papiha S S. Pal B. Gc (vitamin D binding protein) subtypes in rheumatoid arthritis. Hum Genet 1985; 70: 278-80.

28 Harpending H C. Jenkins T. Genetic distance among south African populations. In: Crawford MH, Workman PL, eds. Method and theories of anthropological genetics. University of New Mexico Press, 1973: 177-99.

29 Manthorpe R. Nielsen L S, Petersen S H, Prause J V. Lewis blood group frequency in patients with primary Sjögren's syndrome. Scand J Rheumatol 1985; 14: 159-62. 